\title{
X-rays from Core-collapse Supernovae
}

\author{
David Pooley \\ Department of Physics, Sam Houston State University, Huntsville, Texas 77341 \\ and Eureka Scientific, Inc. \\ email: dave@shsu.edu
}

\begin{abstract}
Core-collapse supernovae can produce X-rays through a variety of mechanisms, which are briefly reviewed. Through a combination of targeted searches of specific supernovae and archival searches for serendipitous coverage of supernovae, the number of known X-ray supernovae has grown by a factor of five in the past 13 years, when the Chandra X-ray Observatory and XMM-Newton were launched. The Swift satellite has contributed greatly to the discovery of X-ray emitted supernovae, but care must taken with all Swift detections given its spatial resolution and the number of X-ray binaries typically seen in external galaxies. About half of the reported Swift detections of X-ray emission from supernovae are in fact not due to the supernovae but from unrelated nearby sources in the host galaxies.
\end{abstract}

Keywords. supernovae: general; stars: mass loss

\section{Introduction}

Over thirty years ago, X-rays were discovered in the direction of supernova (SN) 1980K (Canizares et al. 1982) in the near aftermath (years) of its discovery, marking it the first supernova detected in X-rays at such a young age. The number of additional young supernovae detected in X-rays grew very slowly in the ensuing decade and a half, totaling nine known X-ray emitting supernovae before the launch of the Chandra X-ray Observatory and XMM-Newton in 1999.

In the past 13 years, the number of X-ray supernovae has grown by more than a factor of five to over 50 because of the combined efforts of Chandra, XMM, and the Swift satellite, which was launched in 2004. Figure 1 shows the growth of the known $\mathrm{X}$-ray supernova population as a function of time. Even with the current generation of very powerful X-ray satellites, only a handful of new X-ray supernova detections are made each year. The sensitivity of a typical X-ray observation may be on the order of

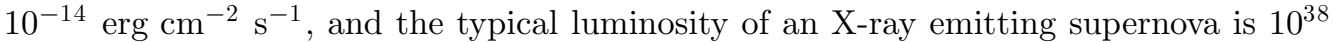
$-10^{39} \mathrm{erg} / \mathrm{s}$, constraining this field of study to the very local universe (Figure 1).

\section{X-ray Production Mechanisms}

Core-collapse supernovae start with a burst of X-rays as the shock breaks out from the dying star, seen for the first time in SN 2008D (Soderberg et al. 2008; Modjaz et al. 2009). The timescale of this event is difficult to explain in a spherically symmetric explosion, but aspherical, jet-driven supernova simulations reproduce both the spectrum and lightcurve of SN 2008D reasonable well (Couch et al. 2011). After the shock breakout, strong X-ray emission is seen again at days to months after the explosion, and in some cases persists for years and even decades.

The X-ray emission of type II supernovae is convincingly explained as thermal radiation $(k T \lesssim 10 \mathrm{keV})$ from the "reverse shock" region that forms within the expanding SN ejecta as it interacts with the dense stellar wind of the progenitor star. The interaction of a 

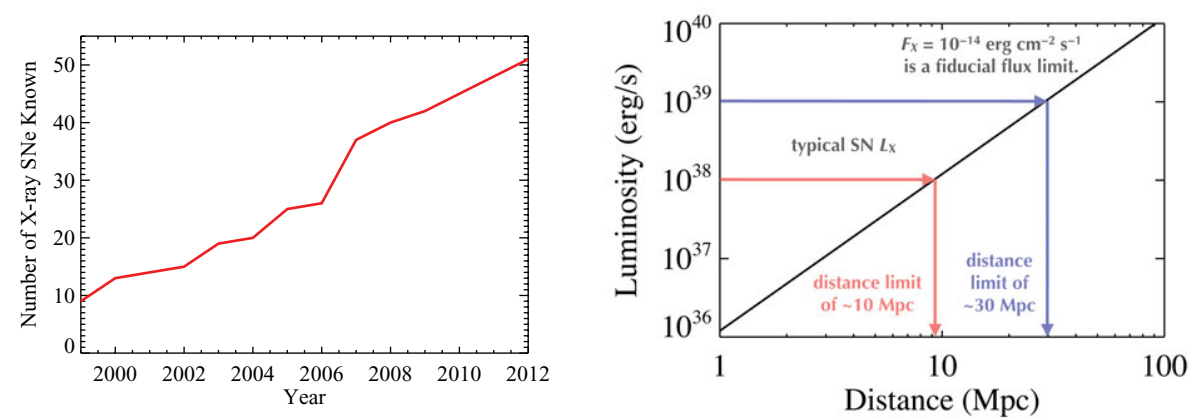

Figure 1. Left: The number of young supernovae known to emit X-rays as a function of time since the launch of Chandra, XMM, and Swift. Right: The typical X-ray luminosity of a supernova is between $10^{38}-10^{39} \mathrm{erg} / \mathrm{s}$, and the typical sensitivity of an X-ray observation is $10^{-14} \mathrm{erg}^{-2} \mathrm{sm}^{-1}$, limiting most observations to within $30 \mathrm{Mpc}$.

spherically symmetric SN shock and a smooth CSM has been calculated in detail (Chevalier 1982a,b; Chevalier \& Fransson 1994; Suzuki \& Nomoto 1995; Fransson et al. 1996). As the supernova shock emerges from the star, its characteristic velocity is $\sim 10^{4} \mathrm{~km} / \mathrm{s}$, and the density distribution in the outer parts of the ejecta can be approximated by a power-law in radius, $\rho \propto r^{-n}$, with $7 \lesssim n \lesssim 20$. The outgoing shock propagates into the dense circumstellar material (CSM) formed by the pre-supernova stellar wind. For red giant progenitors, this wind is slow $\left(v_{w} \sim 10 \mathrm{~km} / \mathrm{s}\right)$ and results from a high mass loss rate $\left(\dot{M} \sim 10^{-4}-10^{-6} M_{\odot} /\right.$ yr $)$. The density for such a wind follows $\rho=\dot{M} / 4 \pi r^{2} v_{w}$. The collision between supernova ejecta and CSM also produces a "reverse" shock, which travels outward at $\sim 10^{3} \mathrm{~km} / \mathrm{s}$ slower than the fastest ejecta. Interaction between the outgoing shock and the CSM produces a hot shell $\left(\sim 10^{9} \mathrm{~K}\right)$, while the reverse shock produces a denser, cooler shell $\left(\sim 10^{7} \mathrm{~K}\right)$ with much higher emission measure from which most of the observable X-ray emission arises. Within this framework of CSM interaction, Chevalier et al. (2006) have shown how X-ray and radio measurements of type IIP SNe
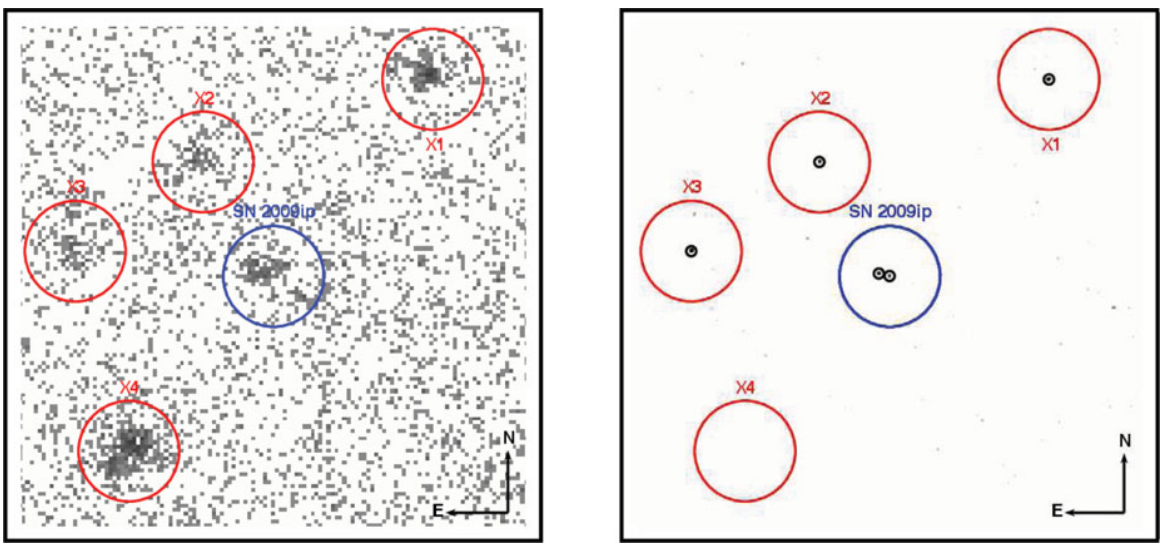

Figure 2. Left: Swift XRT image of the field around SN 2009ip, which is indicated with a blue circle. Nearby sources are indicated by red circles. All circles in this image are $1^{\prime}$ in diameter. Right: Chandra image of the same field, revealing an unrelated point source about $6^{\prime \prime}$ from 2009 ip which is unresolved from it in the Swift XRT image. Black circles indicate Chandra sources and are $6^{\prime \prime}$ in diameter. Note the extreme variability of source X4 between the Swift and Chandra observations; such behavior is typical of X-ray binaries, the types of sources expected to be present around the sites of core-collapse supernovae. 

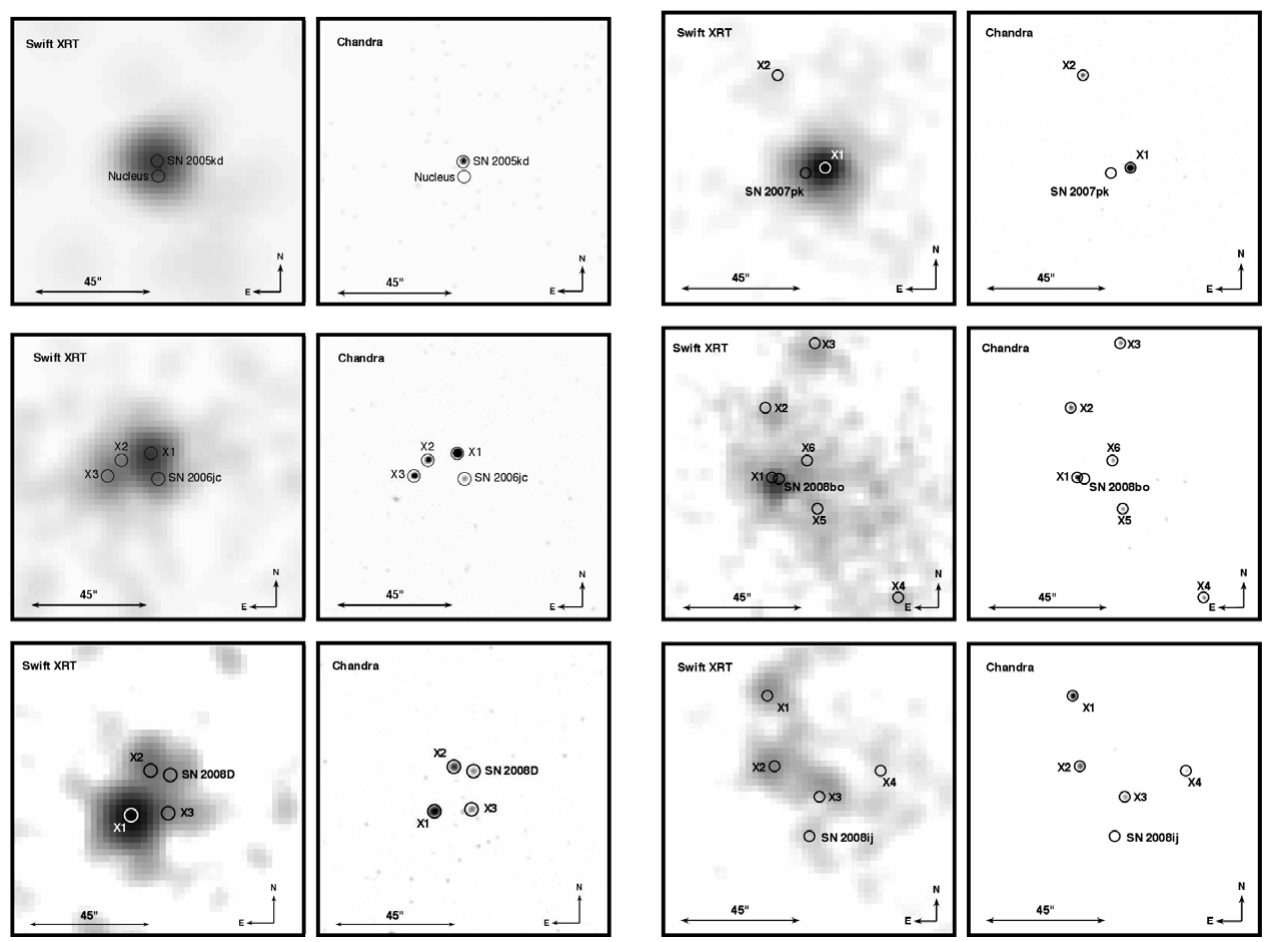

Figure 3. Swift XRT and Chandra images of 2005kd, 2006jc, and 2008D (left) and 2007pk, 2008 bo, and $2008 \mathrm{ij}$ (right). Each image is $\sim 1.8^{\prime}$ on a side. In all cases, there is strong X-ray emission around the site of the supernova as seen with the Swift XRT. In the supernovae on the left, some amount of the flux is attributable to the SN (although contaminated by the flux from nearby sources). However, in the cases on the right, none of the supernovae were detected with Chandra, and all of the Swift XRT flux is due to nearby sources.

are excellent probes of the mass loss of the progenitor star. Dwarkadas \& Gruszko (2012) give an excellent review of CSM interaction and discuss the expected temporal evolution of the X-ray luminosity.

The origin of X-rays from Ib/c supernovae is less clear; the interaction of the supernova shock with the low-density CSM around Ib/c progenitors results in a reverse shock which is too weak to explain the observed X-rays. Chevalier \& Fransson (2006) have suggested an inverse-Compton and synchotron mechanism for the production of X-rays from these supernovae.

The type IIn SNe are perhaps the least understood but can be the most X-ray luminous subtype. The narrow optical lines that characterize the IIn subclass are clear evidence of dense circumstellar gas; they probably arise from reprocessing of X-ray emission. The $\mathrm{X}$-ray emission could result from the shocked ejecta, as in the case of the normal type II $\mathrm{SNe}$, or it could originate from shocked clumps of gas in the CSM (e.g., Chugai 1993). These two scenarios predict vastly different widths for X-ray emission lines, but we have not yet obtained an X-ray spectrum of sufficient quality to make the distinction. For the case where the emission comes from the shocked ejecta, Nymark et al. (2006) have shown the complexity of the resulting X-ray spectrum (e.g., see their Fig. 11) and the dangers of using single-temperature spectral models. Their calculations reveal the rich emission-line spectrum and temperature profile of the radiative shocks. Unfortunately, 
Table 1. Results of Chandra followup of reported Swift X-ray detections.

\begin{tabular}{cc}
\hline $\begin{array}{c}\text { Swift XRT } \\
\text { detection }\end{array}$ & $\begin{array}{c}\text { Chandra } \\
\text { confirmation }\end{array}$ \\
\hline SN 2007pk & $\boldsymbol{x}$ \\
SN 2008bo & $\boldsymbol{x}$ \\
SN 2008ij & $\boldsymbol{x}$ \\
CSS080928 & $\boldsymbol{J}$ \\
SN 2009if & $\boldsymbol{J}$ \\
SN 2009mk & $\boldsymbol{x}$ \\
\hline
\end{tabular}

\begin{tabular}{cc}
\hline $\begin{array}{c}\text { Swift XRT } \\
\text { detection }\end{array}$ & $\begin{array}{c}\text { Chandra } \\
\text { confirmation }\end{array}$ \\
\hline SN 2010jl & $\checkmark$ \\
SN 2010jr & $\checkmark$ \\
SN 2011ay & $\boldsymbol{x}$ \\
SN 2011by & $\boldsymbol{x}$ \\
SN 2011dh & $\sqrt[\checkmark]{ }$ \\
SN 2011ht & $\boldsymbol{x}$ \\
\hline
\end{tabular}

these models have not yet been put in a form usable by standard X-ray spectral fitting packages like Xspec (Arnaud 1996) or Sherpa (Freeman et al. 2001).

\section{Importance of Chandra's Spatial Resolution}

X-ray observations of external galaxies reveal diverse populations of sources, most of which are X-ray binaries ranging in luminosities from below $10^{36} \mathrm{erg} / \mathrm{s}$ to above $10^{41} \mathrm{erg} / \mathrm{s}$ with a variety of spectral shapes and variability on timescales from minutes to decades.

New X-ray emitting supernovae are thus expected to occur in the vicinity of several other X-ray sources, and this is indeed seen. Unfortunately, the Swift X-ray Telescope (XRT), which has proven invaluable in obtaining X-ray light curves of dozens of supernovae, lacks the spatial resolution necessary to associate unambiguously X-ray emission in the direction of a young supernova with the supernova itself. For this, Chandra is necessary, with its spatial resolution over 30 times better than the Swift XRT. A recent example of the necessity of such resolution is SN 2009ip, in which the X-rays seen by Swift XRT are due to both 2009ip and an unrelated source about $6^{\prime \prime}$ away (Figure 2).

For the past five years, my colleagues and I have been awarded Chandra observations of new Swift XRT supernova detections with the aim of determining whether the X-ray emission is due to the supernova or to nearby sources (or both). We have followed up two or three supernovae a year, and Table 1 lists results. A checkmark indicates that at least some of the X-ray emission seen by Swift XRT is due to the supernova, and an X-mark indicates that all of the X-rays seen by the XRT are due to unrelated sources. Less than half of the Swift XRT detections are in fact X-ray emitting supernovae.

\section{References}

Arnaud, K. A. 1996, Astronomical Data Analysis Software and Systems V, 101, 17

Canizares, C. R., Kriss, G. A., \& Feigelson, E. D. 1982, ApJ, 253, L17

Chevalier, R. A. 1982a, ApJ, 258, 790

Chevalier, R. A. 1982 b, ApJ, 259, 302

Chevalier, R. A. \& Fransson, C. 1994, ApJ, 420, 268

Chevalier, R. A., Fransson, C., \& Nymark, T. K. 2006, ApJ, 641, 1029

Chevalier, R. A. \& Fransson, C. 2006, ApJ, 651, 381

Chugai, N. N. 1993, ApJ, 414, L101

Couch, S. M., Pooley, D., Wheeler, J. C., \& Milosavljević, M. 2011, ApJ, 727, 104

Dwarkadas, V. V. \& Gruszko, J. 2012, MNRAS, 419, 1515

Fransson, C., Lundqvist, P., \& Chevalier, R. A. 1996, ApJ, 461, 993

Freeman, P., Doe, S., \& Siemiginowska, A. 2001, Proc. SPIE, 4477, 76

Modjaz, M., Li, W., Butler, N., et al. 2009, ApJ, 702, 226

Nymark, T. K., Fransson, C., \& Kozma, C. 2006, A\&SA, 449, 171 
Pooley, D. 2007, Supernova 1987A: 20 Years After, 937, 381

Sell, P. H., Pooley, D., Zezas, A., et al. 2011, ApJ, 735, 26

Soderberg, A. M., Berger, E., Page, K. L., et al. 2008, Nature, 453, 469

Suzuki, T. \& Nomoto, K. 1995, ApJ, 455, 658

\section{Discussion}

VINK: Has the inverse Compton emission ever been used in conjunction with the radio to estimate the magnetic fields?

Pooley: Yes, see Sayan Chakraborti's paper and the next talk by Alak Ray.

ChAndra: How far away is the x-ray contaminating source in SN 2009ip field?

PoOley: About 6-7"

MoRIYA: Are X-ray faint SNe IIn faint even in optical? Are there any correlations between X-ray luminosity and optical luminosity in SNe IIn.

PoOley: I have not yet tried to correlate X-ray luminosity with optical luminosity for IIn SNe, but that is something I plan to do in the future. 